\title{
Parâmetros psicométricos de Raven - escala avançada série I - para crianças e adolescentes de Curitiba
}

\author{
Raven's psychometric parameters - advanced scale series I - for children and teenagers from Curitiba
}

Plinio Marco de Toni ${ }^{[a]}$, Carla Cristina Reis de Assis Melo ${ }^{[b]}$

\footnotetext{
${ }^{[a]}$ Doutor em Psicologia Escolar e do Desenvolvimento Humano pela Universidade de São Paulo, professor do dep. de Psicologia da Universidade Estadual do CentroOeste (Unicentro), PR - Brasil, email:

pliniomarco@yahoo.com.br

${ }^{[b]}$ Psicóloga graduada pela Universidade Tuiuti do Paraná, Curitiba, PR - Brasil, email: carlaa_melo@yahoo.com.br
}

Recebido: $13 / 05 / 2013$ Received: 05/13/2013

Aprovado: 07/10/2013 Approved: 10/07/2013

\section{Resumo}

A padronização, validade e precisão são parâmetros psicométricos fundamentais no desenvolvimento de instrumentos psicológicos. Sendo assim, o presente estudo teve como objetivo normatizar as Matrizes Progressivas Raven - Escala Avançada - série I, para a população infanto-juvenil do município de Curitiba. Este subtipo é composto por doze itens e cada item do caderno constitui-se de desenhos com uma parte faltante, possibilitando apenas uma resposta correta. $\mathrm{O}$ indivíduo é solicitado a completá-lo, escolhendo entre oito alternativas a que ele acredita ser a correta. A resposta única para aquele item é marcada na folha de respostas. A amostra foi composta por 604 participantes de 10 a 17 anos (media de 13,5 anos), sendo que $52,6 \%$ foram do gênero masculino e $91,2 \%$ destros. Além disso, $65,7 \%$ foram alunos de escolas públicas. Os resultados apontaram que houve diferença significativa entre alunos de escola pública e particular, bem como entre alunos com até um ano de reprovação e de dois a quatro anos. 0 escore dos participantes no teste aumentou em função da idade. Não foram verificadas diferenças significativas entre os gêneros.

Palavras-chave: Padronização. Validade. Precisão

\section{Abstract}

Standardization, validity and precision are fundamental psychometric parameters in developing psychological instruments. Therefore, the aim of the present study is to standardize the Raven's Progressive Matrices -Advanced Scale - Series I, for children and tenagers who live in Curitiba, Brazil. This subtype is comprised of twelve items and each item of the notebook consists of drawings, each one with a missing piece, making possible only one correct response. The subject is prompted to complete it, choosing between eight alternatives to which he believes to be the correct response. The single answer for that item is marked on the answer sheet. The sample was comprised of 604 participants, ranging from 10 to 17 years of age (median age of 13.5 years), $52.6 \%$ of them being of the male gender and $91.2 \%$ being right-handed. Furthermore, $65.7 \%$ were public school students. The results indicated a significant difference between public and private school students, as well as between students who had been retained once and those who had been retained 2 to 4 times. The score of the participants in the test increased in direct correlation with the age of the participants. No significant differences had been noted between genders

Keywords: Standardization. Validity. Precision 


\section{Introdução}

O Teste das Matrizes Progressivas de Raven foi desenvolvido por John C. Raven na Universidade de Dumfries, na Escócia, sendo padronizado e publicado em 1930 (BANDEIRA; ALVES; GIACOMEL; \& LORENZATTO, 2004). É um teste de inteligência criado para abranger todas as faixas de desenvolvimento intelectual, desde o momento em que a criança é capaz de compreender a ideia de encontrar a parte que falta para completar um desenho. Vários autores, entre os quais, Pasquali (1998), Noronha e Vendramini (2003) e Anastasi e Urbina (2000) enfatizam a importância de estudos que garantam a precisão, validade e padronização dos testes psicológicos de forma que todos estejam dentro do padrão de fidedignidade e confiabilidade para serem utilizados. A revisão periódica e a adaptação cultural também se fazem necessário para melhores resultados e mais precisos, como afirma Avancini, Assis, Santos e Oliveira (2005). Este estudo vem complementar e somar com todo o processo de padronização de testes psicológicos, visto que Matrizes Progressivas de Raven vem com um propósito de avaliar a inteligência do indivíduo através de provas que executem seu raciocínio lógico.

Os testes de Raven (RAVEN, 1988) constituem um dos métodos utilizados para medir a inteligência de um indivíduo. 0 teste de inteligência mais útil deve medir uma variedade de habilidades e capacidades (KAPLAN \& SADOK, 1993). Para Paín (1992), as matrizes de Raven se mostram distintas de outros instrumentos de avaliação de inteligência. A escala avançada apresenta ao indivíduo uma tarefa única sob forma de figuras, as matrizes, enquanto outros testes utilizam provas e jogos buscando uma série de comportamentos a partir de estimulações, situações e instruções variadas. 0 teste Matrizes Progressivas de Raven é conhecido no Brasil como Escala Geral e abrange todas as faixas do desenvolvimento intelectual. A sua centralidade indica que não é apenas uma boa medida de inteligência, mas também que a teoria do processamento no teste Raven deve-se ao uso do raciocínio e nos outros testes no centro do espaço, como explicam Carpenter, Just e Shell (1990). Bandeira, Costa E Anteche (2008) colocam que este teste pode ser utilizado em crianças pequenas e com deficiência mental dentro da faixa etária de 5 a 11 anos e também em pessoas idosas. 0 teste envolve o pensamento abstrato, indução de relações e deduções (CARPENTER et al., 1990). A Escala Geral foi planejada para abranger todas as faixas de desenvolvimento intelectual, desde o momento em que a criança é capaz de compreender a ideia de encontrar o pedaço que falta para completar um desenho. (ANGELINI et al., 1999, citado por BANDEIRA et al., 2004). Para Luft, Sanches, Mazo e Andrade (2007), não é recomendável enquadrar os resultados dos testes em categorias como baixo, médio e alto, pois essas categorias podem rotular as crianças como deficientes, normais ou superdotadas, o que pode levar à perda da precisão dos dados. As Matrizes Progressivas de Raven - Escala Superior (RAVEN, 1988) podem ser aplicadas em adolescentes e adultos com nível cultural médio ou elevado, para avaliar a inteligência geral; as matrizes coloridas são aplicadas para crianças e adultos com nível cultural mais baixo, com a mesma finalidade. 0 instrumento possui um caderno com desenhos com uma parte faltante, no qual o indivíduo escolhe entre seis alternativas a resposta que ele acredita ser correta. Sisto, Rueda e Bartholomeu (2006) analisam que, conforme consta no manual do teste, este tem validade mesmo com o aumento da idade cronológica. A validação está relacionada ao instrumento e não ao uso que se faz dos seus escores. Assim, não faz sentido dizer que um teste com validade de construto é válido em uma situação, e não o é em outra (PASQUALI, 2007). Este mesmo autor considera a validade de um teste como primordial (NORONHA et al., 2003). Noronha e Vendramini (2003) testaram alguns testes de Raven, utilizando como critério de avaliação as informações presentes nos manuais, se houve padronização brasileira ou apenas tradução, validade e precisão e comparando-os com testes de personalidade. Concluiu-se que, em geral, há muitas falhas na questão de informação e coerência nos manuais dos instrumentos, e que há muito mais estudos acerca de testes de inteligência, dando uma ênfase maior sobre validade e precisão destes instrumentos. Conforme a Resolução 25/2001 do CFP, "os testes devem ter requisitos mínimos, precisam ser revisados a cada dez anos e seus respectivos manuais devem ter por objetivo orientar o profissional, inclusive na confecção de documentos e relatórios." (NORONHA \& VENDRAMINI, 2003). A padronização também envolve algumas peculiaridades, como procedimentos de aplicação, direitos dos testados, controle das variáveis e normas na divulgação dos resultados, as quais garantem a boa qualidade na coleta dos dados, seu uso adequado e eficaz e também conclusões coerentes, (NORONHA et al. 2003). Rosseti, Rabelo, Leme, Pacanaro e Guntert (2009) 
indicam que as Matrizes progressivas avançadas de Raven apresentam poucos estudos de evidência de validade e precisão para a população brasileira, como destacado pelo CFP em 2007. Para Grassi-Oliveira, Stein e Pezzi (2006) e Schulz, Rossignoli, Correr, Fernadez-Llimos e Toni (2008), a padronização de testes estrangeiros inclui sua tradução para o português e uma retradução para a sua língua original, com o objetivo de verificar se algum termo ou conceito foi perdido. Realiza-se também uma avaliação semântica com profissionais da área estudada e população alvo, a fim de que o significado geral do teste se mantenha e não se limite a um significado referencial. Contudo, ainda "é necessário adaptar a linguagem do ponto de vista cultural e conceitual, buscando aproximá-la ao máximo da realidade da população de interesse." (SCHULZ et al., 2008). Avancini et al. (2005) também enfatizam essa necessidade de uma adaptação transcultural com ajustes regionais. Mesmo que estes testes já tenham a eficácia comprovada, é necessária uma readaptação. (HUTZ \& NUNES, 2001, citado por VASCONCELLOS \& HUTZ, 2008). Pasquali (2001) apresenta que a aplicação de testes é uma das técnicas mais apropriadas para diagnosticar um determinado problema, visando uma possível intervenção.

Carpenter et al. (1990) acreditam que o teste de Raven fornece uma base de teste apropriado para estudos nas inteligências analíticas. Seu formato é um recurso de pesquisa comum por ter um formato não verbal e muito utilizado pelo exército dos países ocidentais. (BELMONT \& MAROLLA, 1973, citados por CARPENTER et al., 1990). Okano, Loureiro, Linhares e Marturano (2004) avaliaram estatisticamente os dados do teste de Raven com a finalidade de verificar a homogeneidade dos grupos estudados quanto ao nível intelectual. A revisão das Matrizes Progressivas - Escala colorida foi utilizada como parâmetro para estabelecer a validade de critério nas pesquisas de padronização de vários testes. Entre eles, podem ser citados a Escala de Maturidade Mental Colúmbia (ALVES \& DUARTE, 1993), o Teste de Inteligência Não Verbal para Crianças - R2 (OLIVEIRA, ROSA \& ALVES, 2000) e a Escala Wechsler de Inteligência para Crianças - WISC III (FIGUEIREDO, 2002; BANDEIRA et al., 2004). Diante disto, viu-se a necessidade de desenvolver um estudo de normatização e precisão das Matrizes Progressivas Raven - Escala Avançada Série I para a população infanto-juvenil do município de Curitiba.

\section{Métodos}

A amostra foi composta por 603 alunos da rede pública estadual e particular de Curitiba, sendo 318 meninos e 285 meninas com idade entre 10 e 18 anos. Deste total, $65,8 \%$ estudavam na rede pública estadual e $34,2 \%$ na rede particular de ensino. A maioria deles, 429 , relatam não terem sido reprovados nenhuma vez. Os instrumentos utilizados foram cadernos de questões contendo 12 itens, um em cada folha. Cada item do caderno constitui-se de desenhos com uma parte faltante, possibilitando apenas uma resposta correta. 0 indivíduo é solicitado a completá-lo, escolhendo entre oito alternativas a que ele acredita ser a correta. A resposta única para aquele item será marcada na folha de respostas, que deve conter os dados de identificação do participante, escolaridade, gênero, índice de reprovação e crivo de correção.

Após a coleta dos termos de consentimento livre e informado, as Matrizes Progressivas Raven - Escala Avançada - Série I foram aplicadas aos participantes da pesquisa de forma coletiva, em ambiente escolar, com uma média de 25 participantes por aplicação. A coleta por turma teve duração de aproximadamente 40 minutos.

Após a coleta dos dados, os desempenhos dos participantes nas Matrizes Progressivas Raven foram analisados por meio de gráficos de intervalo de confiança da média, estatística descritiva e correlação de Pearson, considerando alfa de 5\%.

\section{Resultados}

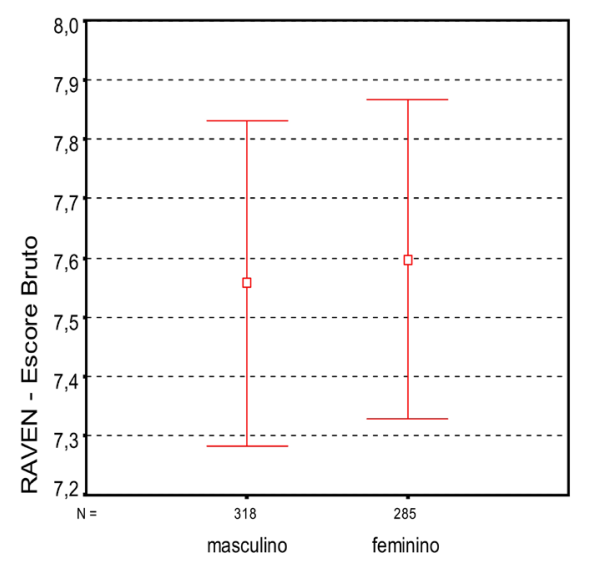

Gráfico 1 - Desempenho dos participantes com relação ao gênero 
O Gráfico 1 compara o desempenho dos participantes nas Matrizes Progressivas Raven - Escala Avançada - Série I em relação ao gênero. 0 resultado demonstra que não houve diferença significativa no escore bruto entre a amostra feminina e masculina, sendo a média próxima de 7,55 para os participantes do gênero masculino e 7,6 para os participantes do gênero feminino.

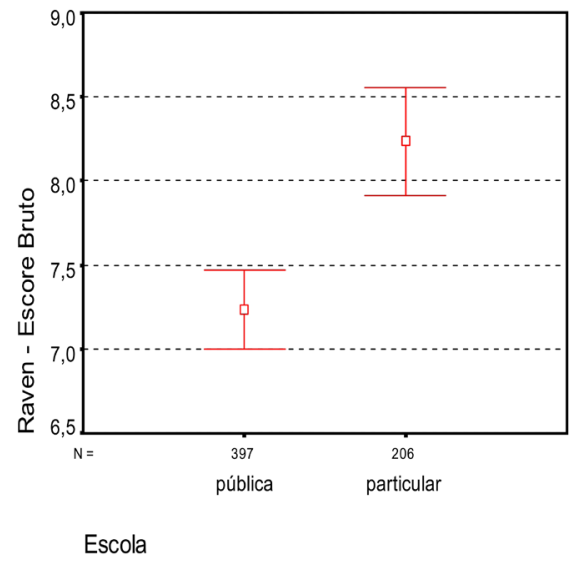
Gráfico 2 - Desempenho dos participantes em relação à escola pública ou
particular

O Gráfico 2 demonstra o desempenho dos participantes em relação à escola, pública ou particular. De acordo os dados apresentados, nota-se que os participantes de escola pública obtiveram uma média de acertos de 7,3, e os participantes da rede particular alcançaram a média de 8,3 no escore total.

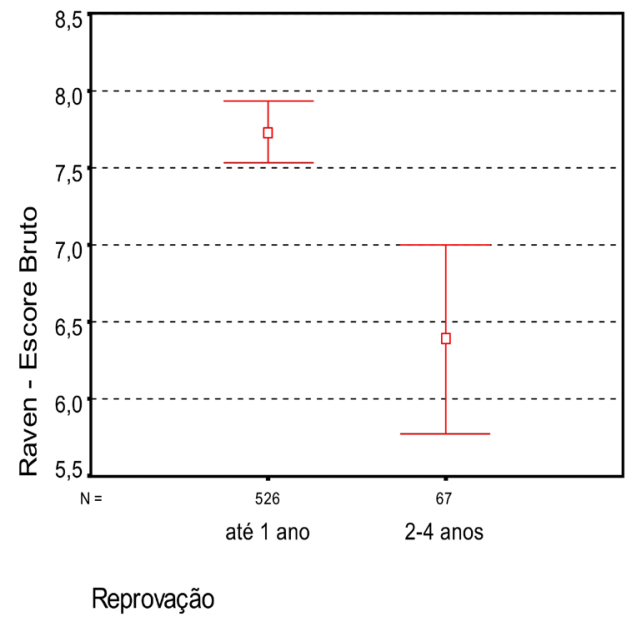

Gráfico 3 - Desempenho dos participantes quanto ao nível ao nível de reprovaç̃o
O Gráfico 3 revela o desempenho dos participantes quanto ao nível de reprovação. Os participantes que relataram não ter tido reprovação ou que já haviam sido reprovados em até um ano, alcançaram média de 7,8 no resultado, enquanto que os participantes com índice de reprovação maior que dois anos obtiveram média de 6,4.

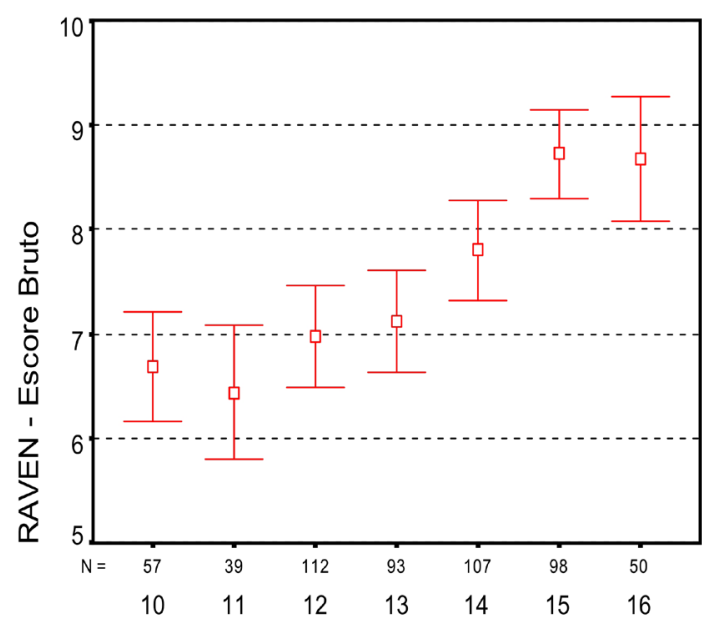

Idade (anos)

Gráfico 4 - Desempenho dos participantes em comparacão com suas idades

O Gráfico 4 compara as idades dos participantes em relação ao seu desempenho. 0 resultado demonstrado apresenta a média de acertos dos participantes com relação à sua idade, demonstrando uma média crescente à medida que a idade dos participantes aumenta.

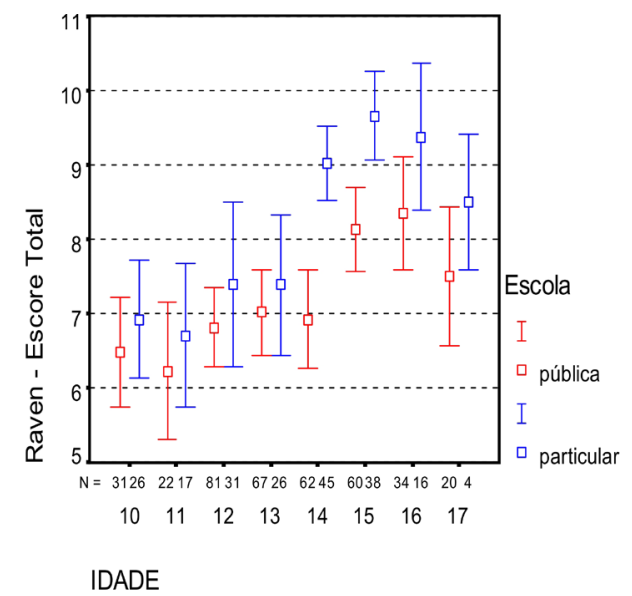

Gráfico 5 - Desempenho dos participantes com relação à idade e rede de escola a que pertencem 
O Gráfico 5 relaciona o desempenho dos participantes referente à idade e tipo de escola. Comparase o desenvolvimento dos participantes tanto da rede pública quanto particular, com relação a suas idades, demonstrando também ligeira diferença no escore obtido na aplicação das Matrizes Progressivas Raven - Escala Avançada - Série I. Nesta figura, também acompanha-se o crescimento das médias de acordo com o aumento da idade dos participantes.

Tabela 1 - Precisão para 0 Teste Matrizes Progressivas Raven - Escala Avançada - Série I

\begin{tabular}{lll}
\hline & Correlações de Pearson & \\
\hline \multirow{3}{*}{ Itens pares } & & Itens ímpares \\
& Correlação de Pearson & 0,58 \\
& Significância (p) & $<0,001$ \\
\hline
\end{tabular}

A Tabela 1 descreve a precisão do teste Raven Escala Avançada - Série I e apresenta o índice de precisão das questões para o Teste Matrizes Progressivas Raven - Escala Avançada - Série I.

Tabela 2 - Tabela de Normatização para o Teste Matrizes Progressivas Raven - Escala Avancada - Série I

\begin{tabular}{lcc}
\hline N & Validade & $\mathbf{6 0 3}$ \\
\hline Média & & 7,58 \\
Desvio Padrão & & 2,41 \\
Mínimo & & 0 \\
Máximo & & 12 \\
Percentis & 1 & 2,00 \\
& 5 & 3,00 \\
& 10 & 4,00 \\
& 25 & 6,00 \\
& 50 & 8,00 \\
& 75 & 9,00 \\
& 90 & 10,00 \\
& 95 & 11,00 \\
& 99 & 12,00 \\
\hline
\end{tabular}

A Tabela 2 é a demonstração da tabela de normatização para as Matrizes Progressivas Raven
- Escala Avançada - Série I. Conforme demonstrado, segue com avaliação dos escores alcançados em relação ao quociente de inteligência, para as Matrizes Progressivas Raven - Escala Avançada - Série I, sendo que para total de acertos 2 obtém-se o percentil 1 ; para 6 acertos, percentil 25; e para 12 acertos, percentil 99.

\section{Discussão dos dados}

Analisando o Gráfico 1 constatou-se que não houve diferença significativa na média de acertos entre os participantes masculinos e femininos. Para Dal'Igna (2007), essa diferença pode ou não existir, mas não relaciona-se à distinção de sexos, e sim à diferença de personalidades de cada um. Em contrapartida, Alves (2006), em seu estudo, verificou diferença entre os gêneros aplicando as Matrizes Progressivas Raven - Escala avançada - Série I.

Percebeu-se uma ligeira diferença no escore bruto do Gráfico 2, que classifica o desempenho dos participantes de acordo com a instituição, pública ou particular, com média de 1 ponto a mais para os participantes que estudam em escola particular. DalVesco et al. (1998) analisaram o rendimento escolar dos alunos de escolas públicas e particulares utilizando o WISC. Os escores apresentados revelam uma diferença significativa entre as duas escolas, sendo mais elevadas as pontuações de alunos da rede particular. A maior concentração do QI total dos alunos da escola particular localiza-se na classificação Muito Superior (70\%), enquanto os da pública se concentraram na classificação Médio Superior (60\%). Verificou-se também essa diferença na Gráfico 5.

0 Gráfico 3 demonstra o desempenho dos participantes em relação ao nível de reprovação, ficando claro que os participantes que tiveram até uma reprovação obtiveram maior escore que os participantes que relataram ter reprovado duas ou mais vezes. Em um estudo realizado por Gatti, Patto, Costa, Kopit e Almeida (1981), constatou-se que não houve variáveis significativas na investigação psicológica com testes de inteligência e avaliações físicas entre alunos recorrentes de reprovação.

0 Gráfico 4 analisou o desenvolvimento dos participantes de acordo com suas idades. Ele mostra que quanto maior a idade do participante testado, maior o número de acertos, ressaltando o que 
Piaget descreve no desenvolvimento da criança e do adolescente. Faria (2001), de acordo com Piaget, apresenta que dos 12 aos 16 anos, a principal tarefa do adolescente é construir as operações formais próprias da inteligência formal. Depois dos 16 anos, não são desenvolvidos novos sistemas mentais, uma vez que as operações formais consolidam-se em sistemas e vão ser utilizadas para o resto da vida.

Na Tabela 1 foi apresentado o índice de precisão das Matrizes Progressivas Raven - Escala Avançada - Série I. Noronha (2003) destaca que os elementos de validade e precisão de testes psicológicos são indispensáveis para garantir a confiabilidade dos resultados das aplicações. Pasquali (2001) complementa que precisão, fidedignidade, constância, consistência interna, confiabilidade, estabilidade, confiança e homogeneidade devem ser analisadas juntas. Anastasi \& Urbina (2000) afirmam que para a verificação da precisão, considera-se a constância dos resultados dos sujeitos em diferentes situações de aplicação ou com aplicações advindas de testes similares. A ausência de estudos desta natureza impede a verificação da manutenção dos resultados e da precisão do teste.

Segundo Pasquali (1998), o termo precisão, quando usado em psicometria, sempre significa estabilidade ou consistência. Precisão do teste é a consistência dos resultados obtidos pelo mesmo indivíduo, quando retestado com o mesmo teste, ou com uma forma equivalente. Para Anastasi \& Urbina (2000), antes de um teste psicológico ser apresentado para o uso geral, é preciso realizar uma verificação completa e objetiva de sua precisão, o que foi demonstrado na Tabela 1.

Noronha \& Vendramini (2003) analisaram alguns testes de inteligência e personalidade para avaliá-los quanto à presença de informações contidas nos manuais, se estavam dentro dos padrões de validade e precisão. Dentre eles está o Teste Matrizes Progressivas de Raven - Escala Avançada.

Para Pasquali (2001) e Noronha e Vendramini (2003), a normatização é um dos parâmetros psicométricos mais importantes para a garantia da qualidade psicométrica de uma medida e se mostra, particularmente, significativa a uma devida interpretação dos escores obtidos por um sujeito no teste. A forma mais comum de comunicação das normas intragrupo é por meio de percentis, que corresponde à divisão de um conjunto ordenado em cem partes iguais, e mediante os escores-padrão, conjunto de processos que comparam as notas brutas individuais com a média do grupo, sendo a média avaliada em unidades de desvio-padrão. $\mathrm{Na}$ Tabela 2 apresenta-se o quadro da normatização com os percentis de cada questão avaliada.

\section{Considerações Finais}

Este estudo teve como objetivo verificar a precisão e normatização das Matrizes Progressivas Raven - Escala Avançada - Série I. Para tal, foi necessária a aplicação do teste em 604 participantes com idade de 10 a 17 anos, tanto de escola pública quanto particular do município de Curitiba. Os resultados obtidos apontaram diferenças significativas no índice de inteligência entre participantes de escola pública e particular e entre alunos que reprovaram até um ano e de dois a quatro anos. Não houve diferença significativa entre os gêneros. Analisando por idade, o resultado obtido varia e aumenta de acordo com a variação das idades dos participantes. Pôde-se estabelecer a tabela de precisão e normatização para as Matrizes Progressivas Raven - Escala Avançada - Série I. Futuras pesquisas nas Matrizes Progressivas Raven - Escala Avançada - Serie I devem ser realizadas, a fim de estudar a validade do teste e estender todo o processo para a Série II, finalizando todo o teste. Anastasi e Urbina (2000) e Pasquali (2001) ressaltam que todos os testes psicológigos devem ser testados em precisão e validade, para gerar um índice de fidedignidade. Noronha e Vendramini (2003) enfatizam a importância da revisão e análise de testes periodicamente para atualizar manuais e documentos com objetivo de melhor orientar cada profissional. Rosseti et al. (2009) também constataram a precisão do instrumento Matrizes Progressivas Raven - Escala Avançada para avaliar aspectos da inteligência. A ausência de estudos desta natureza impede a verificação da manutenção dos resultados e da precisão do teste. Sugere-se um novo estudo para avaliar precisão e normatização das Matrizes Progressivas Raven Escala Avançada - Série II e um estudo de validade para todas as Matrizes Progressivas Raven, bem como um estudo de adaptação cultural, visto que a sociedade brasileira é muito heterogênea, podendo surgir diferentes desempenhos na aplicação, mas tendo, hoje, apenas um único escore determinado. 


\section{Referências Bibliográficas}

Alves, I. C. B. (2006). Novos estudos psicométricos do Teste D70. Avaliação Psicológica, 5(2), 251-3.

Alves, I. C. B. \& Duarte, J. L. M. (1993). Escala de Maturidade Mental Colúmbia: Manual para avaliação e interpretação. São Paulo: Casa do Psicólogo.

Anastasi, A. \& Urbina, S. (2000). Testagem psicológica. Porto Alegre: ArtMed.

Avancini, J.; Assis, S.; Santos, N. C.; \& Oliveira, R. (2005). Escala de violência psicológica contra adolescentes. Revista de Saúde Pública, 39(5)

Bandeira, D. R.; Alves, I. C. B.; Giacomel, A. E.; \& Lorenzatto, L. (2004). Matrizes progressivas coloridas de Raven escala especial: normas para Porto Alegre. Psicologia em estudo, 9(3), 479-86.

Bandeira, D. R.; Costa, A.; \& Arteche, A. (2008). Estudo de Validade do DFH como medida de desenvolvimento cognitivo infantil. Psicologia Reflexão e Crítica, 21(2), 332-37.

Carpenter, P. A; Just, M. A.; \& Shell, P. (1990). What one intelligence test measures: a theoretical account of the processing in the Raven Progressive Matrices test. Psycological Review, 97(3), 404-31.

Dal'Igna, M. C. (2007). Desempenho escolar de meninos e meninas: há diferença? Educação em revista, 46, 241-67.

Dalvesco, A. et al. (1998) Correlação entre WISC e rendimento escolar na escola pública e na escola particular. Psicologia: Reflexão e Crítica, 11(3)

Faria, A. R. (2001). O desenvolvimento da criança e do adolescente segundo Piaget. São Paulo: Atica.

Figueiredo, V. L. M. (2002). Adaptação e Padronização Brasileira da Escala de Inteligência Wechsler para Crianças, Terceira Edição - WISC-III. São Paulo: Casa do Psicólogo.

Gatti, B. A.; Patto, M. H.; Costa, M. L; Kopit, M.; \& Almeida, R. de M. (1981). A reprovação na primeira série do primeiro grau: um estudo de caso. São Paulo: Caderno Pesquisa de São Paulo.

Grassi-Oliveira, R.; Stein, L. M.; \& Pezzi, J. C. (2006). Tradução e validação de conteúdo da versão em português do Childhood Trauma Questionnaire. Revista de Saúde Pública, 40(2), 249-55.
Kaplan, H. I. \& Sadoc, B. J. (1993). Compêndio de Psiquiatria: ciências comportamentais, psiquiatria clínica. Porto Alegre: Artes Médicas Sul.

Luft, C. di B.; Sanches, S. de O.; Mazo, G. Z.; \& Andrade, A. (2007). Versão brasileira da escala de estresse percebido: tradução e validação para idosos. Revista de Saúde Pública, 41(4).

Noronha, A. P. P. (2003). Estudos de validade e de precisão em testes de inteligência. Paidéia, 13(26), 163-9.

Noronha, A. P. P. et al. (2003). Propriedades psicométricas apresentadas em manuais de testes de inteligência. Psicologia em estudo, 8(1), 93-9

Noronha, A. P. P. \& Vendramini, C. M. M. (2003). Parâmetros psicométricos: estudo comparativo entre testes de inteligência e de personalidade. Psicologia: Reflexão e crítica, 16(1), 177-82.

Noronha, A. P. P.; Freitas, F. A.; \& Ottati, F. (2003). Análise de instrumentos de avaliação de interesses profissionais. Psicologia; teoria e pesquisa, 19(3), 287-91.

Okano, C. B.; Loureiro, S. R.; Linhares, B. M.; \& Marturano, E. M. (2004). Crianças com dificuldades escolares atendidas em programa de suporte psicopedagógico na escola: avaliação do autoconceito. Psicologia: Reflexiva e Crítica, 17(1), 121-8

Oliveira, R.; Rosa, H. R.; Alves, I. C. B. (2000). R-2: Teste Não Verbal de Inteligência para Crianças - Manual. São Paulo: Vetor.

Paín, S. (1992). Psicometria genética. São Paulo: Casa do Psicólogo.

Pasquali, L. (1998). Psicometria: teoria e aplicação. Brasilia: UNB.

Pasquali, L. (2001). Técnicas de exame psicológico (TEP) Manual: fundamentos das técnicas psicológicas. Vol. 1. São Paulo: Casa do Psicólogo.

Pasquali, L. (2007). Validade dos testes psicológicos: será possível reencontrar o caminho? Psicologia: Teoria e pesquisa, 23, 99-107.

Raven, J. \& Raven, J. C. (1988). Matrizes Progressivas Coloridas de Raven - Manual. São Paulo: Casa do Psicólogo.

Rosseti, M. O.; Rabelo, I. S.; Leme, I. A. de S.; Pacanaro, S. V.; \& Guntert, I. B.(2009). Evidências de qualidade das Matrizes Progressivas Avançadas de Raven em universitários. São Paulo: Psico USF. 
Schulz, R. B.; Rossignoli, P.; Correr, C. J., Fernandez-Llimos, F.; \& Toni, P. M. de. (2008). Validação de mini questionário de qualidade de vida em hipertensão arterial (Minichal) para o português (Brasil). Arquivos Brasileiros de Cardiologia, 90(2)
Sisto, F. F.; Rueda, F. J. M.; \& Bartholomeu, D. (2006). Estudo sobre a unidimensionalidade do teste Matrizes Progressivas Coloridas de Raven. Psicologia: Reflexão e Crítica, 19(1), 66-73.

Vasconcellos, S. J. L. \& Hutz, C. S. (2008). Construção e validação de uma escala de abertura à experiência. Avaliação Psicológica, 7(2), 135-41 